\title{
Finding New Forces with Old Techniques
}

\author{
Interactions predicted by beyond-standard-model theories could be \\ detected using a variation on Mössbauer spectroscopy, according to a new \\ proposal.
}

By Marric Stephens

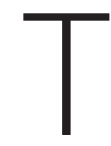
he number of known fundamental interactions has been stuck at four since the discovery of the strong force in the 1970s. However, some beyond-standard-model theories predict additional interactions that might be detectable over submicrometer scales. Giorgio Gratta, at Stanford University, and colleagues now propose a way to measure such forces by exploiting a well-known technique-Mössbauer spectroscopy [1].

When a nucleus emits a gamma-ray photon in free space, it recoils. The recoil energy means that the emitted photon has an energy lower than that of a photon that could be absorbed by the same transition. The Mössbauer effect occurs when nuclei are bound within a solid matrix: with the recoil suppressed, the emitted photon carries more of the energy. In a Mössbauer spectrometer, this effect is used to match the spectra of a gamma-ray source and of an absorber. If the chemical environment of nuclei in the absorber perturbs the nuclei's

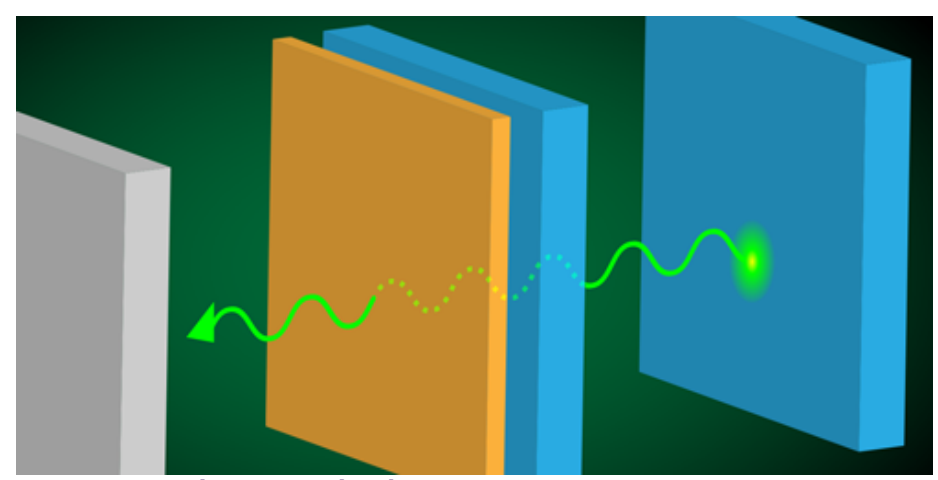

Credit: APS/Alan Stonebraker energy levels, it causes a measurable spectral mismatch between source and absorber.

Gratta and colleagues propose to use this principle to look for energy-level perturbations caused not by the chemical environment but by new fundamental interactions. The scheme involves bringing a dense solid, such as gold or frozen xenon, to within micrometers of the absorber. Via the hypothesized interactions, nuclei in this solid would shift the absorber's energy levels, producing potentially detectable gamma-ray-absorption changes. The technique would be especially sensitive to scalar interactions, which might alter nuclear energy levels by causing changes to quark masses or to the fine-structure constant. The researchers say the experiment could be done with available technology as soon as labs fully reopen after the lockdowns.

Marric Stephens is a Corresponding Editor for Physics based in Bristol, UK.

\section{REFERENCES}

1. G. Gratta et al., "Searching for new interactions at submicron scale using the Mössbauer effect," Phys. Rev. D 102, 115031 (2020). 